\title{
Review
}

\section{Squamous-cell vulval cancer}

\author{
Kanishka Karunaratne ${ }^{1}$ \\ Sri Lanka Journal of Obstetrics and Gynaecology 2011; 33: 30-32
}

\section{Introduction}

Vulval cancer is a rare condition, accounting for $1 \%$ to $2 \%$ of all female genital tract malignancies in Sri Lanka. Figures are slightly higher in the Western World accounting for about $4 \%$. The condition is more prevalent in women over 65 years of age. In recent years an increased incidence of vulval cancers in younger women has been observed in the UK, mainland Europe and the USA. In the UK the incidence in younger women has doubled over the past three decades ${ }^{1}$. Squamous-cell carcinomas account for more than $90 \%$ of vulval cancers. The other $10 \%$ includes melanomas, sarcomas, basal-cell carcinomas and adenocarcinomas.

\section{Precursor lesions in vulval cancer}

Human Papilloma Virus (HPV) is reported as present in approximately $40 \%$ of vulval carcinomas, with HPV 16 accounting for most HPV-positive cases². In older women, keratinizing squamous-cell vulval carcinomas are more common, with the majority found to be HPV-negative. Conversely, in younger women, basaloid squamous- cell vulval carcinomas are more common where the prevalence of HPV is high, especially when associated with vulval intraepithelial neoplasia $(\mathrm{VIN})^{2}$. The increased incidence of vulval cancer worldwide is believed to be linked to an increasing incidence of VIN in younger women caused by HPV infections. Two different types of VIN are recognized. Undifferentiated VIN is related to HPV infections and differentiated VIN is related to chronic inflammatory skin disease.

\section{Vulval intraepithelial neoplasia}

VIN is a precancerous condition of the vulval skin, most commonly presenting with localized vulval pruritus. The classification of VIN is based upon the International Society for the Study of Vulvovaginal Disease (ISSVD) terminology, where VIN is classified as VIN warty type, VIN basaloid type, VIN mixed type (warty and basaloid) and VIN differentiated type ${ }^{2}$. Warty or basaloid VIN is more common in younger

\footnotetext{
${ }^{1}$ Consultant Gynae-oncologist, National Cancer Institute, Maharagama, Sri Lanka.

E-mail:kushank@sltnet.lk
}

premenopausal women. Differentiated VIN is more common in older women and often found accompany lichen sclerosus ${ }^{3}$. If untreated VIN may remain stable, spontaneously regress or progress to vulval carcinoma. The mean interval between the diagnosis of VIN 3 and invasion in untreated cases has been reported as four years ${ }^{4}$.

\section{Lichen sclerosus}

Lichen sclerosus is a lymphocyte-mediated dermatosis commonly present in the anogenital region of post-menopausal women. Although of somewhat uncertain aetiology, autoimmune or hormonal mechanisms are suggested. Lichen sclerosus has a 3$5 \%$ risk of progression to vulval cancer and in older women, squamous-cell vulval carcinomas is often associated with lichen sclerosus and squamous hyperplasia ${ }^{5}$.

Women with VIN and/or lichen sclerosus are often managed by a dermatologist or gynaecologist with a special knowledge in vulval cancer ${ }^{6}$. Risk factors for vulval cancers are illustrated in Box 1.

\section{Squamous-cell vulval cancer}

Women with vulval cancer may present with a vulval lesion or pruritis and pain. Presentation with bleeding is not uncommon and examination of the vulva in women reporting postmenopausal bleeding is crucial. When there is a background history of VIN or lichen sclerosus attention should be paid to worsening symptoms or persisting symptoms that remain unresponsive to treatment.

\section{Investigations}

With above symptoms one should undergo a vulval biopsy to detect cancer at the earliest opportunity. HPV related other cancer sites (cervix, anus) should be thoroughly examined ${ }^{7,8}$. In early vulval tumours, the risk of lymph node metastasis is reported as low. Clinical assessment of the lymph node alone is not recommended ${ }^{9}$. Systematic review, however, suggest that ultrasound, computerised tomography (CT), magnetic resonance imaging (MRI), positron emission tomography (PET) and fine-needle biopsy show inconsistent results and are not accurate enough for routine assessment of groin nodes status ${ }^{9}$. 


\section{Box 1. Risk factors for vulval cancer}

Pre-invasive lesions

- Vulval intraepithelial neoplasia

- Lichen sclerosus

Other anogenital cancer or family history of anogenital cancer

- Previous high-grade cervical intraepithelial neoplasia/cervical cancer

- Vaginal cancer

- Anal cancer

- Upper aerodigestive squamous-cell carcinoma

Social

$$
\text { - Smoking }
$$

Sexually transmitted infections

- Genital warts/human papilloma virus

- Herpes simplex virus type II

- HIV

Immunocompromise

- Renal transplant

- HIV

Other medical conditions

- Systemic lupus erthymatosus

\section{Sentinel lymph node assessment}

The gold standard for detection of nodal metastasis in vulval cancer remains histological evaluation of the surgically removed lymph nodes. Sentinel node identification is currently the most promising diagnostic tool for assessment of lymph node status in vulval cancer. Radio-labelled colloid in conjunction with a marking dye is used for the detection of the sentinel node, which is the first lymph node receiving lymphatic drainage from the tumour. The aim of sentinel node biopsy is to establish the presence of groin node metastasis in squamous-cell vulval cancer and therefore reduce the need for radical groin lymphadenectomy. The International Sentinel Node Society has recommended performing sentinel node biopsy only in patients with tumours $<4 \mathrm{~cm}$ with procedure on the whole recommended for early stage tumours only. Sentinel node biopsy needs to be adequately validated in prospective clinical trials. The results of Gynaecologic Oncology Group study (GOG173 , study of intraoperative lymphatic mapping in patients with invasive squamous-cell carcinoma) are awaited.

\section{Staging of vulval cancers}

Staging is only really achievable following surgical excision and through pathological examination of the primary tumour and corresponding lymph nodes. Staging is performed using the International Federation of Gynaecology and Obstetrics (FIGO) system. The most vulval cancers present as in situ or early-stage disease. Thirty per cent of patients are reported as having lymph node metastasis at presentation.

\section{Treatment of vulval cancers}

Treatment for vulval cancer, like in many diseases, needs to be individualized, and done at a multidisciplinary oncology unit. Primary surgery followed by radiotherapy (if required) is the mainstay of treatment. However, large tumours or tumours located at urethral or anal orifices can be treated with primary chemo-radiation followed by surgery.

\section{Surgical management of vulval cancer}

In more recent years surgery for vulval cancer has become less radical in an aim to reduce the debilitating side-effects and complications (Box 2). Microinvasion (FIGO-1A) can be managed by wide local tumour excision only ${ }^{10,11}$. Radical vulvectomy for early vulval cancers has now been replaced by wide local excision which appears to be a safe alternative as the recurrence rate is low ${ }^{12}$. The traditional butterfly incision has been replaced by the triple incision technique ${ }^{12}$.

Depending on the position of the primary tumour, lymph nodes may be removed either unilaterally or bilaterally. Midline lesions $(<1 \mathrm{~cm}$ from the midline) require bilateral inguinofemoral node dissection. In patients with lateral early-stage tumours omission of contra lateral lymph node dissection appears safe.

\section{Box 2. Surgical morbidity associated with vulval cancer}

- Wound breakdown/dehiscence

- Wound infection

- Deep vein thrombosis and pulmonary embolism

- Vaginal/introital stenosis

- Urinary incontinence/faecal incontinence

- Lymphocyst formation

- Hernia

- Lymphoedema

- Psychosexual dysfunction 


\section{Radiotherapy and chemotherapy}

In early-stage vulval cancer, surgery is the firstchoice treatment for the groin nodes, as groin node irradiation as a primary treatment results in higher recurrence rate ${ }^{13}$. Radiotherapy is more commonly used as an adjuvant treatment following primary surgery. Present UK guideline recommend adjuvant radiotherapy when inadequate surgical margins of excision are obtained or when either groin has two or more lymph nodes involved with microscopic metastatic disease. Radical radiotherapy is used in patients for whom surgery is not an option and is usually combined with chemotherapy. Preoperative radio therapy may allow for shrinkage of primary tumour to achieved sphincter-preservation in patients with tumours involving the urethra and anus.

\section{Follow up}

All women with a diagnosis with vulval cancer should undergo regular post-treatment follow up. Follow up is recommended at 3-monthly interval for the first year, 6-monthly interval for the next two years and then annually.

\section{References}

1. Cancer Research UK. UK vulva cancer incidence statistics, 2008 (http:/ /info.cancerresearchuk.org/cancersats/types/ vulva/incidence/ ?a=5441; accessed 20 August 2009).

2. Smith J, Backes, D,Hoots B, et al. Human papilloma virus type-distribution in vulvar and vaginal cancers and their associated precursors. Obstet Gynaecol 2009; 113: 917-24).

3. Royal College of Pathologists. Dataset for histological reporting of vulval neoplasms, version 2, 2nd edn. London: Royal College of Pathologists, 2008.
4. Jones RW, Rowen DM, Stewart AW. Vulvar intraepithelial neoplasms: aspects of the natural history and outcome in 405 women. Obstet Gynaecol 2005; 106: 1319-26.

5. Neill SM, Tatnall FM, Cox NH. Guidelines for the management of lichen sclerosus. Br J Dermatol 2002; 147: 640-9.

6. Tidy JA, Soutter WP, Luesley DM, et al. Management of lichen sclerosus and intraepithelial neoplasia of the vulva in the UK. J R Soc Med 1996; 89: 699-701.

7. Edgren G, Sparen P. Risk of anogenital cancer after diagnosis of cervical intraepithelial neoplasia: a prospective population-based study. Lancet Oncol 2007; 8: 311-6.

8. Kalliala I, Anttila A, Pukkala E, et al. Risk of cervical and other cancers after treatment of cervical intraepithelial neoplasia: retrospective cohort study. BMJ 2005; 331: 1183-5.

9. Selman TJ, Luesley DM, Acheson N, et al. A systematic review of accuracy of diagnostic tests for inguinal lymph node status in vulval cancer. Gynaecol Oncol 2005; 99: 206-14.

10. Hacker NF. Vulval cancer. In: Berek JS, Hacker NF, eds, Practical Gynaecology Oncology, 4th edn. Philadelphia, PA Lippincott Williams \& Wilkins, 2005.

11. Parker RT, Duncan I, Rampone J, et al. Operative management of early invasive epidermoid carcinoma of the vulva. Am J Obstet Gynecol 1975; 123: 349-54.

12. Ansink A, Stegeman M, van der Veldon $K$, et al. Surgical interventions for early squamous cell carcinoma of the vulva. Cochrane Database Syst Rev 1999, Issue 4. Art. No.: CD002036. DOI:10.1002/14651858.CD002036.

13. Van der Velden K, Ansink A. Primary groin irradiation versus primary groin surgery for early vulval cancer. Cochrane Database Sys Rev 2001, Issue 4, Art. No.:CD002224. DOI: 10.1002/14651858. CD002224. 